\title{
The Association between Job Strain and Atrial Fibrillation: Results from the Swedish WOLF Study
}

\author{
Eleonor I. Fransson, ${ }^{1,2}$ Magdalena Stadin, ${ }^{1}$ Maria Nordin, ${ }^{3,4}$ Dan Malm, ${ }^{1,5}$ \\ Anders Knutsson, ${ }^{6}$ Lars Alfredsson, ${ }^{2}$ and Peter J. M. Westerholm ${ }^{7}$ \\ ${ }^{1}$ School of Health Sciences, Jönköping University, 55111 Jönköping, Sweden \\ ${ }^{2}$ Institute of Environmental Medicine, Karolinska Institutet, 17177 Stockholm, Sweden \\ ${ }^{3}$ Department of Psychology, Umeå University, 90187 Umeå, Sweden \\ ${ }^{4}$ Stress Research Institute, Stockholm University, 10691 Stockholm, Sweden \\ ${ }^{5}$ Department of Internal Medicine, County Hospital Ryhov, 55185 Jönköping, Sweden \\ ${ }^{6}$ Department of Health Sciences, Mid Sweden University, 85170 Sundsvall, Sweden \\ ${ }^{7}$ Occupational and Environmental Medicine, Uppsala University, 75185 Uppsala, Sweden
}

Correspondence should be addressed to Eleonor I. Fransson; eleonor.fransson@hhj.hj.se

Received 16 January 2015; Accepted 23 March 2015

Academic Editor: Giancarlo Cesana

Copyright (C) 2015 Eleonor I. Fransson et al. This is an open access article distributed under the Creative Commons Attribution License, which permits unrestricted use, distribution, and reproduction in any medium, provided the original work is properly cited.

\begin{abstract}
Introduction. Atrial fibrillation (AF) is a common heart rhythm disorder. Several life-style factors have been identified as risk factors for AF, but less is known about the impact of work-related stress. This study aims to evaluate the association between work-related stress, defined as job strain, and risk of AF. Methods. Data from the Swedish WOLF study was used, comprising 10,121 working men and women. Job strain was measured by the demand-control model. Information on incident AF was derived from national registers. Cox proportional hazard regression was used to estimate hazard ratios (HR) and 95\% confidence intervals (CI) for the association between job strain and AF risk. Results. In total, 253 incident AF cases were identified during a total follow-up time of 132,387 person-years. Job strain was associated with AF risk in a time-dependent manner, with stronger association after 10.7 years of follow-up (HR 1.93, 95\% CI 1.10-3.36 after 10.7 years, versus HR 1.11, 95\% CI 0.67-1.83 before 10.7 years). The results pointed towards a dose-response relationship when taking accumulated exposure to job strain over time into account. Conclusion. This study provides support to the hypothesis that work-related stress defined as job strain is linked to an increased risk of AF.
\end{abstract}

\section{Introduction}

Cardiovascular disease (CVD) is the leading cause of death in a global perspective. According to the World Health Organization (WHO) 17.3 million people died from CVD in 2008, which represents $30 \%$ of the global deaths [1]. Atrial fibrillation (AF) is the most common cardiac arrhythmia and is also a well-confirmed risk factor of stroke [2-4]. Symptoms of atrial fibrillation include palpitations, shortness of breath, fatigue, chest pain, dizziness, and reduced physical capacity. Atrial fibrillation often affects the patients, as well as their family members, with distress and reduced well-being in their daily life [5-7]. In 2010, the estimated global age-adjusted prevalence of $\mathrm{AF}$ in the population of 35 years and older was
1368.5 per 100000 in men and 856.8 in women [8]. In Europe, $3.7-4.2 \%$ of those aged $60-70$ and $10-17 \%$ of those 80 years or older suffer from AF [9]. The prevalence of AF in Sweden is estimated to be $2.9 \%$ [10]. The incidence and prevalence of AF increase with age [11-14], and AF is more common in men than women $[8,11]$. During the recent decades, the incidence of AF has increased, and this tendency is presumed to maintain [8].

It is not unusual that AF occurs in conjunction with other CVD (e.g., heart failure and heart valve problems) and hypertension [15]. However, AF may also occur without the impact of those factors and hereditary and life style factors are likely to play a part in the pathogenesis of AF [1518]. Obesity, sleep apnea, heavy alcohol consumption, and 
prolonged physical exertion are examples of life-style factors that have been proposed as risk factors of AF [15, 18-22].

Mental stress is often reported by AF patients as a triggering factor of AF attacks [23], but few studies have evaluated the association between psychosocial stress and AF. However, in a case-control study it was found that acute life stress was related to AF risk [24], and in one recently published prospective study, an association between workrelated stress and increased risk of AF was observed [25].

Although AF is common among the general population and considered as a public health disease, the knowledge about different risk factors and AF is still insufficient. The aim of this study is to provide additional knowledge about the relation between work-related stress, defined as job strain, and the onset of AF.

\section{Material and Methods}

Data were obtained from the Work, Lipids, and Fibrinogen (WOLF) study, a longitudinal occupational cohort study conducted in Sweden [26]. The original aim with the WOLF study was to investigate the associations between psychosocial work environment and cardiovascular risk factors.

2.1. Procedure and Participation. The baseline data collection in WOLF was carried out in the county of Stockholm during 1992-1995 (WOLF Stockholm, $n=5698$ ). In 19961998 , the data collection was extended to northern Sweden, in the counties of Västernorrland and Jämtland (WOLF Norrland, $n=4718$ ). WOLF Norrland was partly established in order to recruit more blue-collar workers into the project. Altogether 36 occupational health service units in the counties of Stockholm, Västernorrland, and Jämtland were invited to participate. Of those, 33 occupational health service units accepted to participate. The occupational health service units represented approximately 60 companies in different branches comprising various occupations. However, including all employees at the 60 companies was not possible. Instead, all employees representing certain workplaces (e.g., a department, garage, institution, laboratory, and sales organization) were asked to participate. This selection was basically due to practical reasons from the perspective of the occupational health service units. Employees who were on more or less permanent leave from the workplace, for example, those stationed abroad or chronically ill, were not included in the study population. The participation rate at baseline was $82 \%$, with higher participation rate in the northern part in Sweden.

At baseline, the participants filled in an extensive questionnaire covering different occupational aspects (e.g., work tasks, work hours, and work environment), sociodemographic aspects (e.g., education level), and lifestyle habits (e.g., smoking and physical exercise) as well as different aspects of health. In addition, a minor clinical examination was conducted by specially trained nurses at the occupational health service units. The clinical examination included measurements of height, weight, waist and hip circumference, and blood pressure. Blood samples were also collected.
A follow-up study in WOLF Norrland was conducted in 2000-2003. In total, 3633 participants from WOLF Norrland provided repeated measurements on work and life-style factors by taking part in the follow-up study.

2.2. Analytical Sample. In total, 10416 working men and women participated by answering the questionnaire and taking part of the clinical examination at baseline. For the present study, we excluded participants who reported that they had experienced a myocardial infarction or heart failure prior to baseline. We also excluded those with a recorded diagnosis of AF in national hospital discharge and outpatient registers prior to baseline, leaving 10121 participants (6971 men and 3150 women) as our analytical sample. In the analyses with repeated measurements, 3123 participants were included.

2.3. Work-Related Stress according to the Demand-Control Model. Work-related stress was defined according to Karsek's job demand-control model $[27,28]$, which is characterized by the combination of psychological job demands and control over the work situation. The model proposes that those experiencing high job demands in combination with low control (i.e., high strain or job strain) are in a stressful work situation and are at higher risk for developing ill health. In this study we used the Swedish demand-control questionnaire containing five job demands items and six control items to measure job strain [29]. Cronbach's alpha for the job demands and job control subscales was 0.72 and 0.74 , respectively. Mean response scores for the job demands and for the job control items were computed for each participant. We used the median scores as cut-points for high and low demands ("high demands" being defined as scores strictly above the study-specific median score) and job control ("low control" being defined as scores strictly below the study-specific median score). In the analyses we used both a dichotomized measure of job strain (high strain versus all others) and four categories based on the combination of job demands and control: low strain jobs (low demands, high control); passive jobs (low demands, low control); active jobs (high demands, high control); and high strain jobs (high demands, low control).

2.4. Atrial Fibrillation. Information on incident AF, or flutter, was derived from the Swedish national hospital discharge, outpatient, and mortality registers by using the following ICD codes: ICD-10 code I48; ICD-9 code 427D; and ICD-8 code 427.92 .

2.5. Potential Confounding and Mediating Factors. In addition to age in years (continuous), sex, and part of study (Stockholm, Norrland), we considered the following factors as potential confounding or mediating factors: socioeconomic status (manual workers, lower level/intermediate nonmanual employees, and professionals), exercise (seldom, sometimes, and regularly), smoking (never (neither current nor ex-smoker), ex-smoker (has previously smoked for at least one year but is not a current smoker), and current 
smoker), alcohol consumption (none (0 units/week), moderate (1-14 units/week for women, 1-21 units/week for men), intermediate (15-20 units/week for women, 22-27 units/week for men), and heavy (21 units/week or more for women, 28 units/week or more for men)), waist circumference $(<94 \mathrm{~cm}$ (men) or $<80 \mathrm{~cm}$ (women), $94-101.99 \mathrm{~cm}$ (men) or $80-88$ (women), and $>102 \mathrm{~cm}$ (men) or $>88 \mathrm{~cm}$ (women)), and hypertension (systolic blood pressure $\geq 140 \mathrm{mmHg}$, or diastolic blood pressure $\geq 90 \mathrm{mmHg}$, or self-reported treatment with antihypertensive drugs).

2.6. Statistical Analyses. The participants were followed up from their baseline assessment of job strain to the first registered AF event, migration out of Sweden, death, or end of follow-up, whichever came first. Independent $t$-tests and $\mathrm{Chi}^{2}$-tests for bivariate analyses were conducted in order to test potential differences in baseline characteristics between participants with and without AF. Cox proportional hazard regression was used to estimate hazard ratios (HR) and 95\% confidence intervals (CI) to quantify the relationship between job strain and risk of atrial fibrillation. All analyses were adjusted for age, sex, and part of study. Other potential confounding and mediating factors were added one by one to the age, sex, and part of study adjusted Cox proportional hazard regression model. Only those factors changing estimates of job strain versus others with more than $10 \%$ were to be included in subsequent models [30]. To evaluate a potential effect modification by sex, a stratified analysis by sex was carried out, as well as including a statistical interaction term between job strain and sex in the Cox proportional hazard regression model. Kaplan-Meier curves were used to inspect the proportionality of hazards over time. Analyses of accumulated exposure to job strain and the risk of $\mathrm{AF}$ were carried out in a subsample of the WOLF Norrland study population for whom repeated measures of job strain were available. In the analyses with repeated measures, the start of follow-up time was set at the date of the second data collection. A $P$ value for trend was derived by including the variable on accumulated job strain as a continuous variable with three levels in the Cox proportional hazard regression model. All data analyses were carried out using SAS version 9.2.

2.7. Ethics. All participants gave informed consent to participate in the study. The WOLF study has been approved by the Ethics Committee at Karolinska Institutet, Stockholm (\# 92198), and the Regional Ethical Review Board in Stockholm (\# 2006/257-31, \# 2008/1638-31/5).

\section{Results}

Characteristics of the study sample are presented in Table 1. During a total follow-up time of 132,387 person-years (median follow-up time 13.6 years), 253 incident AF events were recorded. Compared to participants without AF, participants with AF were more likely to be male, older, and current or ex-smokers and have higher waist circumference and more likely to suffer from hypertension.
TABLE 1: Baseline characteristics in the total study sample and among participants with and without atrial fibrillation (AF), the WOLF study, Sweden.

\begin{tabular}{|c|c|c|c|c|}
\hline Characteristics & $\begin{array}{c}\text { Total } \\
n=10121\end{array}$ & $\begin{array}{c}\text { Not AF } \\
n=9868\end{array}$ & $\begin{array}{c}\text { AF } \\
n=253\end{array}$ & $P$ value* \\
\hline Age, mean (sd) & $42.5(10.7)$ & $42.3(10.7)$ & $51.3(8.3)$ & $<0.001$ \\
\hline \multicolumn{5}{|l|}{ Sex, $n(\%)$} \\
\hline Men & $6971(69)$ & $6757(68)$ & $214(85)$ & \multirow{2}{*}{$<0.001$} \\
\hline Women & $3150(31)$ & $3111(32)$ & $39(15)$ & \\
\hline \multicolumn{5}{|l|}{ Study part, $n(\%)$} \\
\hline Stockholm & $5518(55)$ & $5368(54)$ & $150(59)$ & \multirow{2}{*}{0.12} \\
\hline Norrland & $4603(45)$ & $4500(46)$ & $103(41)$ & \\
\hline \multicolumn{5}{|l|}{$\begin{array}{l}\text { Demand-control, } n \\
(\%)\end{array}$} \\
\hline No strain & $8960(89)$ & $8739(89)$ & $221(87)$ & \multirow{3}{*}{0.55} \\
\hline Job strain & $1161(11)$ & $1129(11)$ & $32(13)$ & \\
\hline Low strain & $3192(32)$ & $3112(32)$ & $80(32)$ & \\
\hline Passive & $3259(32)$ & $3186(32)$ & $73(29)$ & \multirow{3}{*}{0.64} \\
\hline Active & $2509(25)$ & $2441(25)$ & $68(27)$ & \\
\hline High strain & $1161(11)$ & $1129(11)$ & $32(13)$ & \\
\hline \multicolumn{5}{|l|}{ SES, $n(\%)$} \\
\hline Manual workers & $4422(44)$ & $4310(44)$ & $112(44)$ & \multirow{4}{*}{0.09} \\
\hline $\begin{array}{l}\text { Lower level/ } \\
\text { intermediate }\end{array}$ & & & & \\
\hline $\begin{array}{l}\text { nonmanual } \\
\text { employees }\end{array}$ & $4341(43)$ & $4242(44)$ & $99(39)$ & \\
\hline Professionals & $1234(12)$ & $1192(12)$ & $42(17)$ & \\
\hline \multicolumn{5}{|l|}{$\begin{array}{l}\text { Physical exercise, } n \\
(\%)\end{array}$} \\
\hline Seldom & $2488(25)$ & $2420(25)$ & $68(27)$ & \multirow{3}{*}{0.64} \\
\hline Sometimes & $3876(38)$ & $3781(38)$ & $95(38)$ & \\
\hline Regularly & $3727(37)$ & $3639(37)$ & $88(35)$ & \\
\hline \multicolumn{5}{|l|}{ Smoking, $n(\%)$} \\
\hline Never smokers & $4739(48)$ & $4664(48)$ & $75(30)$ & \multirow{3}{*}{$<0.001$} \\
\hline Ex-smokers & $2893(29)$ & $2788(29)$ & $105(42)$ & \\
\hline Current smokers & $2250(23)$ & $2182(23)$ & $68(27)$ & \\
\hline \multicolumn{5}{|l|}{$\begin{array}{l}\text { Alcohol } \\
\text { consumption, } n(\%)\end{array}$} \\
\hline Non & $487(5)$ & $470(5)$ & $17(7)$ & \multirow{4}{*}{0.37} \\
\hline Moderate & $8688(88)$ & $8476(88)$ & $212(87)$ & \\
\hline Intermediate & $318(3)$ & $311(3)$ & $7(3)$ & \\
\hline Heavy & $401(4)$ & $394(4)$ & $7(3)$ & \\
\hline \multicolumn{5}{|l|}{ Waist circumference } \\
\hline $\begin{array}{l}\leq 94.0(\mathrm{M}) ; \leq 80.0 \\
(\mathrm{~W})\end{array}$ & $6322(63)$ & $6211(63)$ & $111(44)$ & \multirow{3}{*}{$<0.001$} \\
\hline $\begin{array}{l}\text { 94-101.99 (M); } \\
80-88(\mathrm{~W})\end{array}$ & $2241(22)$ & $2174(22)$ & $67(26)$ & \\
\hline$>102(\mathrm{M}) ;>88(\mathrm{~W})$ & $1511(15)$ & $1436(15)$ & $75(30)$ & \\
\hline \multicolumn{5}{|l|}{ Hypertension, $n(\%)$} \\
\hline No & $8043(80)$ & $7891(80)$ & $152(60)$ & \multirow{2}{*}{$<0.001$} \\
\hline Yes & $2062(20)$ & $1961(20)$ & $101(40)$ & \\
\hline
\end{tabular}

In the age, sex, and part of study adjusted Cox proportional hazard regression model, job strain was associated with a $38 \%$ increased risk of AF when compared to all others, although the association was not statistically significant (HR 1.38 , 95\% CI 0.95-2.00) (Table 2). None of the investigated potential confounding or mediating factors changed 
TABLE 2: The estimated association between job strain and the risk of atrial fibrillation. Hazard ratios (HR) with 95\% confidence intervals (95\% CI).

\begin{tabular}{lccc}
\hline Work-related stress & $\begin{array}{c}\text { HR (95\% CI) } \\
\text { Complete follow-up, 253 events }\end{array}$ & $\begin{array}{c}\text { HR (95\% CI) } \\
\text { First 10.7 years of follow-up, 165 events }\end{array}$ & $\begin{array}{c}\text { HR (95\% CI) } \\
\text { Follow-up after 10.7 years, 88 events }\end{array}$ \\
\hline No strain & 1 (ref) & 1 (ref) & $1($ ref) \\
Job strain & $1.38(0.95-2.00)$ & $1.11(0.67-1.83)$ & $1.93(1.10-3.36)$ \\
\hline Low strain & $1($ ref $)$ & $1($ ref $)$ & $1($ ref $)$ \\
Passive & $1.08(0.79-1.49)$ & $1.05(0.71-1.54)$ & $1.16(0.66-2.03)$ \\
Active & $1.21(0.87-1.67)$ & $1.22(0.82-1.83)$ & $1.20(0.67-2.09)$ \\
High strain & $1.50(0.99-2.27)$ & $1.19(0.69-2.06)$ & $2.13(1.13-4.04)$ \\
\hline
\end{tabular}

${ }^{*}$ Adjusted for age, sex, and part of study.

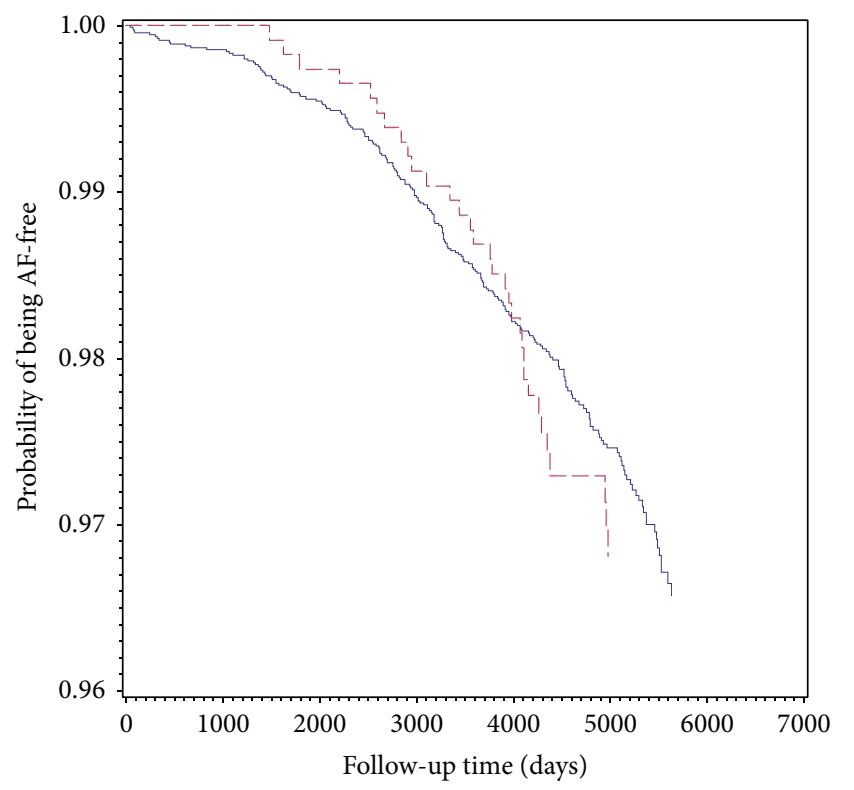

Job strain

- No

-- - Yes

FIgURE 1: Unadjusted Kaplan-Meier plot, job strain versus no strain.

the estimated HR with more than $10 \%$ and were therefore not included in the regression model. No clear effect modification by sex was observed (HR 1.42, 95\% CI 0.94-2.14 for men, HR 1.22, 95\% CI 0.51-2.92 for women, $P$ value for interaction $=0.75)$. When using the four demand-control categories, high strain was associated with a 50\% increased risk of AF compared with the low strain group, the result being borderline significant (HR 1.50, 95\% CI 0.99-2.27) (Table 2).

When inspecting the crude Kaplan-Meier plot, it was observed that those with job strain had a slightly better probability of being AF-free during the first years of follow-up as compared with the nonstrain group, but the curves crossed at approximately 10.7 years after baseline (Figure 1). This led us to do stratified analysis, splitting the follow-up period at 10.7 years after baseline. The seemingly lower AF risk in the job strain group during the first follow-up period was mainly explained by higher prevalence of job strain among women than men (14\% versus $10 \%)$ and that the job strain group
TABLE 3: The estimated association between accumulated exposure to job strain and the risk of atrial fibrillation. Hazard ratios (HR) with $95 \%$ confidence intervals (95\% CI), based on a subsample from the WOLF Norrland study population with baseline measure in 1996-1998 (t1) and repeated measure in 2000-2003 (t2), $n=3123$.

\begin{tabular}{lcc}
\hline & $\begin{array}{c}\mathrm{HR} \\
(95 \% \mathrm{CI})^{*}\end{array}$ & $\begin{array}{c}P \text { value } \\
\text { for trend }\end{array}$ \\
\hline $\begin{array}{l}\text { Subsample with repeated } \\
\text { measurements, } 47 \text { events } \\
\quad \text { Unexposed to job strain at } \\
\text { both t1 and t2 }\end{array}$ & 1 (ref) & 0.06 \\
Job strain at either t1 or t2 & $1.68(0.83-3.40)$ & \\
$\quad$ Job strain at both t1 and t2 & $2.28(0.70-7.44)$ & \\
\hline
\end{tabular}

${ }^{*}$ Adjusted for age and sex.

tended to be younger than the nonstrain group (mean age 41.2 versus 42.7 years). After adjusting for age, sex, and study part, the HR for job strain versus others was 1.11 (95\% CI 0.67-1.83) during the first part of the follow-up period. In the analysis based on the follow-up period after 10.7 years, it was observed that job strain versus all others was significantly associated with the risk of AF in the age, sex, and study part adjusted models (HR 1.93 95\% CI 1.10-3.36) (Table 2). The same pattern was seen when using the four demand-control categories, where the HR for the high strain group compared with low strain was 2.13 (95\% CI 1.13-4.04) (Table 2).

For a subsample of the WOLF Norrland study population we had repeated measurements of job strain, measured at baseline (1996-1998) and follow-up (2000-2003). Taking into account the exposure to job strain at none $(n=2472$, AF cases $=34)$, one $(n=527, \mathrm{AF}$ cases $=10)$, or both measurement occasions ( $n=124$, AF cases $=3$ ), we observed an association between job strain and AF risk in a dose-response manner (Table 3). However, as the number of participants and AF cases exposed at both occasions was few, the estimates were imprecise.

\section{Discussion}

In this study, we observed an association between workrelated stress, defined as job strain, and the risk of atrial fibrillation. The association was time-dependent and more pronounced at the end of the follow-up period. The risk of 
AF was approximately two times higher among those exposed to job strain compared to those unexposed during the latter part of the follow-up period. In analyses taking repeated measurements into account, our results were suggestive of a dose-response relationship between accumulated exposure to job strain and AF risk.

Published studies on the association between psychosocial stress in general and work-related stress in particular and $\mathrm{AF}$ are scarce. We are only aware of one previously published study on job strain and AF risk [25]. That recently published study by Torén et al. was also based on a Swedish sample but only included men and used a job exposure matrix based on occupation at baseline to measure job strain. They found a $32 \%$ increased risk associated with being exposed to job strain versus all others (HR 1.32, 95\% CI 1.003-1.75) [25], which is in accordance with our overall HR estimate of 1.38. In another study, mental stress in terms of acute life stress was found to be related to AF risk [24], and in a study conducted among AF patients, mental stress was the most frequent reported triggering factor of AF attacks [23]. Some case reports linking emotional stress to AF have also been published [31,32]. In addition, different aspects of work-related stress, including job strain, effort-reward imbalance, and job insecurity, have been linked to increased risk of coronary heart disease [3335].

The biological pathway between work-related stress and $\mathrm{AF}$ is not clear. Ectopic foci in pulmonary veins are recognized as triggers of AF, and the processes leading to the onset of AF include atrial fibrosis, structural remodeling of the heart tissue, and inflammation [36]. Altered sympathetic and parasympathetic balance and neurohormonal activation have also been proposed to play key roles in the development of $\mathrm{AF}[36,37]$. In a study on $77 \mathrm{AF}$ patients, Bettoni and Zimmerman showed an increase in adrenergic tone followed by a marked shift towards vagal predominance immediately before the onset of paroxysmal AF [38], and Patterson et al. showed in an experimental study on dogs that both the parasympathetic and sympathetic nervous system have a role in initiating and triggering pulmonary vein activity [39]. Reactions to stress include several physiological responses involving both the hypothalamic-pituitary-adrenal axis and the autonomous nervous system [40,41]. Responses include increased release of glucocorticoid hormones, such as cortisol, and increased sympathetic activity, with increased release of adrenaline and noradrenaline. An effect on inflammation has also been observed [40]. These factors are making a link between psychosocial stress, including work-related stress, and AF plausible. Atrial fibrosis and structural remodeling develop over time and may be asymptomatic for several years. This may explain our finding with a stronger association between job strain and AF observed at the later part of the follow-up period and that accumulated exposure to job strain over time seems to be associated with higher risk as compared to shorter episodes of exposure, although the exact mechanism behind this observation is not clear.

Our study has several strengths, including the prospective design, being based on a large sample of working people, and including both men and women. A high participation rate and low internal dropout are further strengths. We used a well-established measure on work-related stress, based on the demand-control or job strain model, frequently used in studies on work-related stress and health related outcomes. Job strain was measured by self-report through a validated questionnaire [29, 42]. The outcome was defined through national registers with high quality and coverage [43]. One major advantage with our study is that we had access to repeated measures of job strain for a subset of our study sample. We also had access to several potential confounding factors, which we could take into account in the analyses. Indeed, participants diagnosed with AF during the follow-up period were to a higher degree male, older, smoker, and obese and were more likely to suffer from hypertension, which is in accordance with previous studies $[8,11,15,18]$. However, after adjusting for age, sex, and study part, taking into account life-style factors, obesity or hypertension did not change the estimated association in any substantial way. However, it is important to acknowledge that an unfavorable work situation may affect life-style factors in a longitudinal perspective [44], potentially contributing to the association between long-term exposure to job strain and ill health.

Our study also has some limitations. Despite the large study sample and a rather long follow-up period (median follow-up time: 13.6 years), the number of AF cases was relatively low, limiting the power of the study. This is especially true for the longitudinal analyses taking repeated measures into account. Also, the majority of the incident AF cases in our study had an unspecified AF diagnosis, preventing more detailed analyses of AF subtypes such as paroxysmal, persistent, and chronic AF. Furthermore, the experience and perception of stress at work is a complex issue, and there are several ways of operationalizing work-related stress. Here, we used the most frequently utilized model, the job strain model. However, there are several other models and operationalizations available covering other aspects of work-related stress, such as the effort-reward imbalance, job insecurity, and organisational injustice, which is not covered in the present study. Evaluating other aspects of work-related stress in relation to AF in addition to the job strain model will yield a more complete picture of the association between work-related stress and AF.

\section{Conclusion}

Our study lends some support to the hypothesis that workrelated stress, defined as job strain, is related to the development of AF over time. Our results suggest that the association may be time-dependent and that long-term exposure to job strain may be more strongly associated with AF risk than shorter bouts of exposure.

\section{Conflict of Interests}

The authors declare no conflict of interests.

\section{Acknowledgments}

The study has received funding from the Swedish Heart and Lung Association and Futurum, the Academy for Healthcare, Jönköping County Council. 


\section{References}

[1] World Health Organization, Global Atlas on Cardiovascular Disease Prevention and Control, edited by S. Mendis, P. Puska and B. Norrving, World Health Organization, Geneva, Switzerland, 2011.

[2] A. J. Camm, G. Y. H. Lip, R. de Caterina, and et al, "2012 focused update of the ESC Guidelines for the management of atrial fibrillation: an update of the 2010 ESC Guidelines for the management of atrial fibrillation-developed with the special contribution of the European Heart Rhythm Association," Europace, vol. 14, no. 10, pp. 1385-1413, 2012.

[3] E. Z. Soliman, M. M. Safford, P. Muntner et al., "Atrial fibrillation and the risk of myocardial infarction," JAMA Internal Medicine, vol. 174, no. 1, pp. 107-114, 2014.

[4] D. A. Dulli, H. Stanko, and R. L. Levine, "Atrial fibrillation is associated with severe acute ischemic stroke," Neuroepidemiology, vol. 22, no. 2, pp. 118-123, 2003.

[5] T. Dalteg, E. Benzein, A. Sandgren, B. Fridlund, and D. Malm, "Managing uncertainty in couples living with atrial fibrillation," The Journal of Cardiovascular Nursing, vol. 29, no. 3, pp. E1-E10, 2014.

[6] H. Ekblad, H. Rönning, B. Fridlund, and D. Malm, "Patients' well-being: experience and actions in their preventing and handling of atrial fibrillation," European Journal of Cardiovascular Nursing, vol. 12, no. 2, pp. 132-139, 2013.

[7] P. J. McCabe and S. A. Barnason, "Illness perceptions, coping strategies, and symptoms contribute to psychological distress in patients with recurrent symptomatic atrial fibrillation," Journal of Cardiovascular Nursing, vol. 27, no. 5, pp. 431-444, 2012.

[8] S. S. Chugh, R. Havmoeller, K. Narayanan et al., "Worldwide epidemiology of atrial fibrillation: a global burden of disease 2010 study," Circulation, vol. 129, no. 8, pp. 837-847, 2014.

[9] M. Zoni-Berisso, F. Lercari, T. Carazza, and S. Domenicucci, "Epidemiology of atrial fibrillation: European perspective," Clinical Epidemiology, vol. 6, pp. 213-220, 2014.

[10] U. Friberg, P. J. Svensson, and M. von Arbin, Atrial fibrillation. Prevalence and Risk for Stroke, SBU, The Swedish Council on Health Technology Assessment, Stockholm, Sweden, 2013.

[11] A. S. Go, E. M. Hylek, K. A. Phillips et al., "Prevalence of diagnosed atrial fibrillation in adults: National implications for rhythm management and stroke prevention: the anticoagulation and risk factors in atrial fibrillation (ATRIA) study," Journal of the American Medical Association, vol. 285, no. 18, pp. 23702375, 2001.

[12] J. Heeringa, D. A. M. van der Kuip, A. Hofman et al., "Prevalence, incidence and lifetime risk of atrial fibrillation: the Rotterdam study," European Heart Journal, vol. 27, no. 8, pp. 949-953, 2006.

[13] W. B. Kannel, P. A. Wolf, E. J. Benjamin et al., "Prevalence, incidence, prognosis, and predisposing conditions for atrial fibrillation: population-based estimates," The American Journal of Cardiology, vol. 82, no. 8, pp. 2N-9N, 1998.

[14] S. Rietbrock, E. Heeley, J. Plumb, and T. van Staa, "Chronic atrial fibrillation: incidence, prevalence, and prediction of stroke using the congestive heart failure, hypertension, age $>75$, diabetes mellitus, and prior Stroke or transient ischemic attack (CHADS2) risk stratification scheme," The American Heart Journal, vol. 156, no. 1, pp. 57-64, 2008.

[15] B. A. Schoonderwoerd, M. D. Smit, L. Pen, and I. C. van Gelder, "New risk factors for atrial fibrillation: causes of 'not-so-lone atrial fibrillation," Europace, vol. 10, no. 6, pp. 668-673, 2008.
[16] M. F. Sinner, N. R. Tucker, K. L. Lunetta et al., "Integrating genetic, transcriptional, and functional analyses to identify 5 novel genes for atrial fibrillation," Circulation, vol. 130, no. 15 , pp. 1225-1235, 2014.

[17] B. Zöller, H. Ohlsson, J. Sundquist, and K. Sundquist, "High familial risk of atrial fibrillation/atrial flutter in multiplex families: a nationwide family study in Sweden," Journal of the American Heart Association, vol. 2, no. 1, Article ID e003384, 2013.

[18] M. Rosiak, M. Dziuba, M. Chudzik et al., "Risk factors for atrial fibrillation: not always severe heart disease, not always so 'lonely,' Cardiology Journal, vol. 17, no. 5, pp. 437-442, 2010.

[19] D. Conen and C. M. Albert, "Alcohol consumption and risk of atrial fibrillation: how much is too much?" Journal of the American College of Cardiology, vol. 64, no. 3, pp. 290-292, 2014.

[20] S. C. Larsson, N. Drca, and A. Wolk, "Alcohol consumption and risk of atrial fibrillation: a prospective study and dose-response meta-analysis," Journal of the American College of Cardiology, vol. 64, no. 3, pp. 281-289, 2014.

[21] D. Karasoy, T. Bo Jensen, M. L. Hansen et al., "Obesity is a risk factor for atrial fibrillation among fertile young women: a nationwide cohort study," Europace, vol. 15, no. 6, pp. 781-786, 2013.

[22] A. Rosengren, P. J. Hauptman, G. Lappas, L. Olsson, L. Wilhelmsen, and K. Swedberg, "Big men and atrial fibrillation: effects of body size and weight gain on risk of atrial fibrillation in men," European Heart Journal, vol. 30, no. 9, pp. 1113-1120, 2009.

[23] A. Hansson, B. Madsen-Härdig, and S. B. Olsson, "Arrhythmiaprovoking factors and symptoms at the onset of paroxysmal atrial fibrillation: a study based on interviews with 100 patients seeking hospital assistance," BMC Cardiovascular Disorders, vol. 4, article 13, 2004.

[24] A. V. Mattioli, S. Bonatti, M. Zennaro, R. Melotti, and G. Mattioli, "Effect of coffee consumption, lifestyle and acute life stress in the development of acute lone atrial fibrillation," Journal of Cardiovascular Medicine, vol. 9, no. 8, pp. 794-798, 2008.

[25] K. Torén, L. Schioler, M. Soderberg, K. W. Giang, and A. Rosengren, "The association between job strain and atrial fibrillation in Swedish men," Occupational and Environmental Medicine, vol. 72, no. 3, pp. 177-180, 2015.

[26] L. Alfredsson, N. Hammar, E. Fransson et al., "Job strain and major risk factors for coronary heart disease among employed males and famales in a Swedish study on work, lipids and fibrinogen," Scandinavian Journal of Work, Environment and Health, vol. 28, no. 4, pp. 238-248, 2002.

[27] R. A. Karasek, "Job demands, job decision latitude, and mental strain: implications for job redesign," Administrative Science Quarterly, vol. 24, no. 2, pp. 285-308, 1979.

[28] R. Karasek and T. Theorell, Healthy Work: Stress, Productivity and the Reconstruction of Working Life, Basic Books, New York, NY, USA, 1990.

[29] T. Theorell, "The demand-control-support model for studying health in relation to the work environment-an interactive model," in Behavioral Medicine Approaches to Cardiovascular Disease Prevention, K. Orth-Gomér and N. Schneiderman, Eds., pp. 69-85, Lawrence Erlbaum Associates, Mahwah, NJ, USA, 1996.

[30] K. J. Rothman, S. Greenland, and T. L. Lash, Modern Epidemiology, Lippincott Williams \& Wilkins, Philadelphia, Pa, USA, 3rd edition, 2008. 
[31] R. C. Ziegelstein, "Acute emotional stress and cardiac arrhythmias," The Journal of the American Medical Association, vol. 298, no. 3, pp. 324-329, 2007.

[32] D. Legallois, S. Gomes, A. Pellissier, and P. Milliez, "Medical emotional stress-induced atrial fibrillation: my own personal experience," International Journal of Cardiology, vol. 167, no. 6, pp. e182-e183, 2013.

[33] M. Kivimäki, M. Virtanen, M. Elovainio, A. Kouvonen, A. Väänänen, and J. Vahtera, "Work stress in the etiology of coronary heart disease-a meta-analysis," Scandinavian Journal of Work, Environment and Health, vol. 32, no. 6, pp. 431-442, 2006.

[34] M. Kivimäki, S. T. Nyberg, G. D. Batty et al., "Job strain as a risk factor for coronary heart disease: a collaborative meta-analysis of individual participant data," The Lancet, vol. 380, no. 9852, pp. 1491-1497, 2012.

[35] M. Virtanen, S. T. Nyberg, G. D. Batty et al., "Perceived job insecurity as a risk factor for incident coronary heart disease: systematic review and meta-analysis," British Medical Journal, vol. 347, Article ID f4746, 2013.

[36] B. Aldhoon, V. Melenovský, P. Peichl, and J. Kautzner, "New insights into mechanisms of atrial fibrillation," Physiological Research, vol. 59, no. 1, pp. 1-12, 2010.

[37] P. Taggart, M. R. Boyett, S. Logantha, and P. D. Lambiase, "Anger, emotion, and arrhythmias: from brain to heart," Frontiers in Physiology, vol. 2, article 67, 2011.

[38] M. Bettoni and M. Zimmermann, "Autonomic tone variations before the onset of paroxysmal atrial fibrillation," Circulation, vol. 105, no. 23, pp. 2753-2759, 2002.

[39] E. Patterson, S. S. Po, B. J. Scherlag, and R. Lazzara, “Triggered firing in pulmonary veins initiated by in vitro autonomic nerve stimulation," Heart Rhythm, vol. 2, no. 6, pp. 624-631, 2005.

[40] A. Hänsel, S. Hong, R. J. A. Cámara, and R. von Känel, "Inflammation as a psychophysiological biomarker in chronic psychosocial stress," Neuroscience and Biobehavioral Reviews, vol. 35, no. 1, pp. 115-121, 2010.

[41] A. Steptoe and M. Kivimäki, "Stress and cardiovascular disease," Nature Reviews Cardiology, vol. 9, no. 6, pp. 360-370, 2012.

[42] H. S. Chungkham, M. Ingre, R. Karasek, H. Westerlund, and T. Theorell, "Factor structure and longitudinal measurement invariance of the demand control support model: an evidence from the Swedish Longitudinal Occupational Survey of Health (SLOSH)," PLoS ONE, vol. 8, no. 8, Article ID e70541, 2013.

[43] J. F. Ludvigsson, E. Andersson, A. Ekbom et al., "External review and validation of the Swedish national inpatient register," $B M C$ Public Health, vol. 11, article 450, 2011.

[44] E. I. Fransson, K. Heikkila, and S. T. Nyberg, "Job strain as a risk factor for leisure-time physical inactivity: an individualparticipant meta-analysis of up to 170,000 men and women: the IPD-work consortium," American Journal of Epidemiology, vol. 176, no. 12, pp. 1078-1089, 2012. 


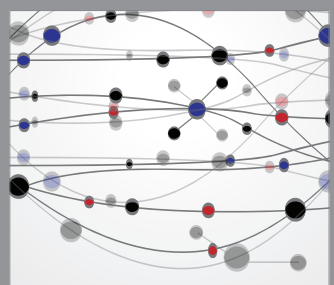

The Scientific World Journal
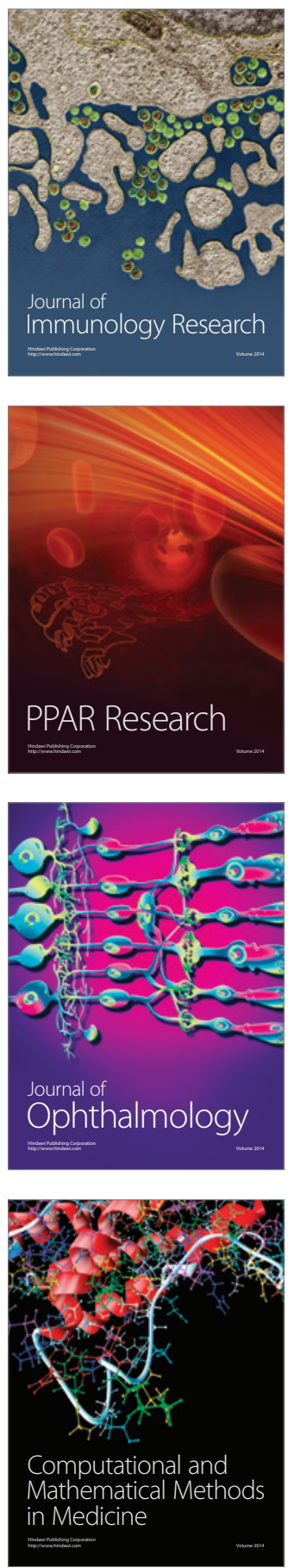

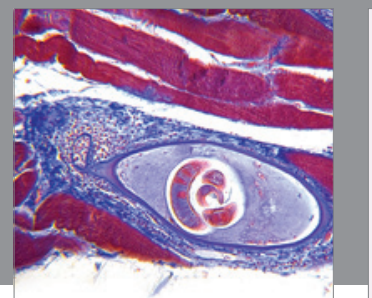

Gastroenterology

Research and Practice
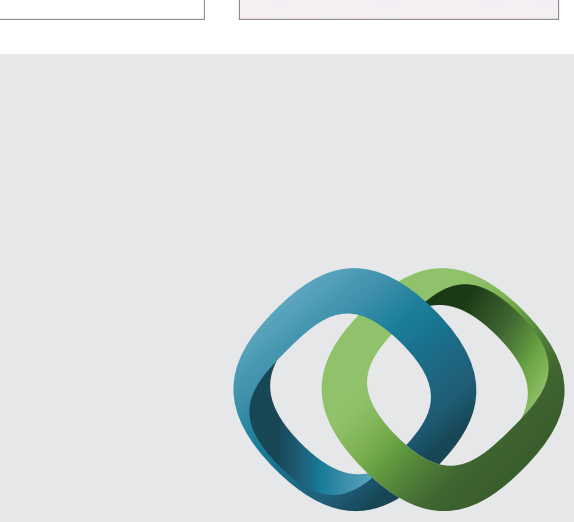

\section{Hindawi}

Submit your manuscripts at

http://www.hindawi.com
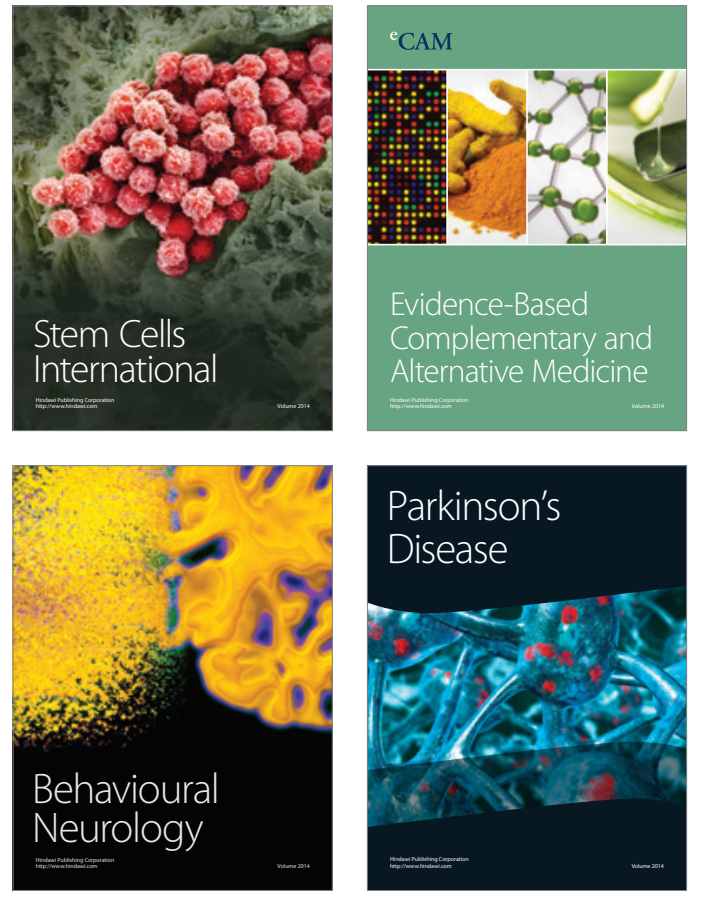
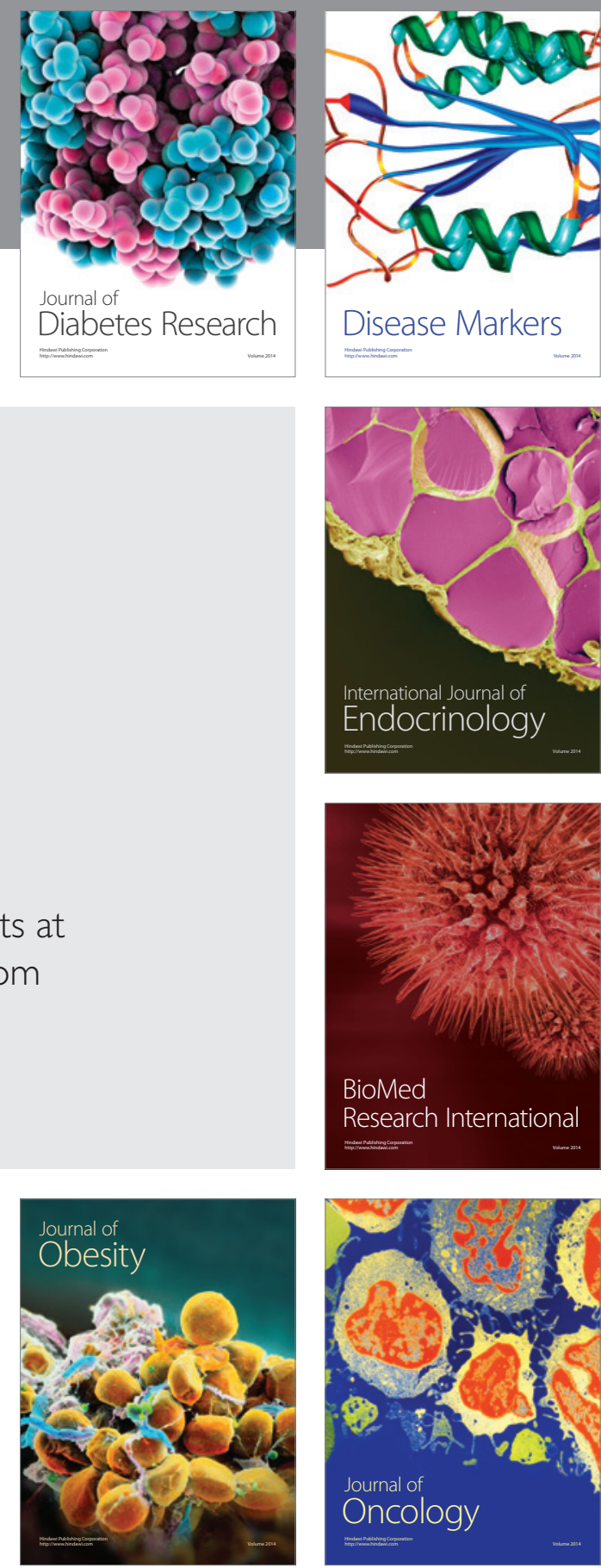

Disease Markers
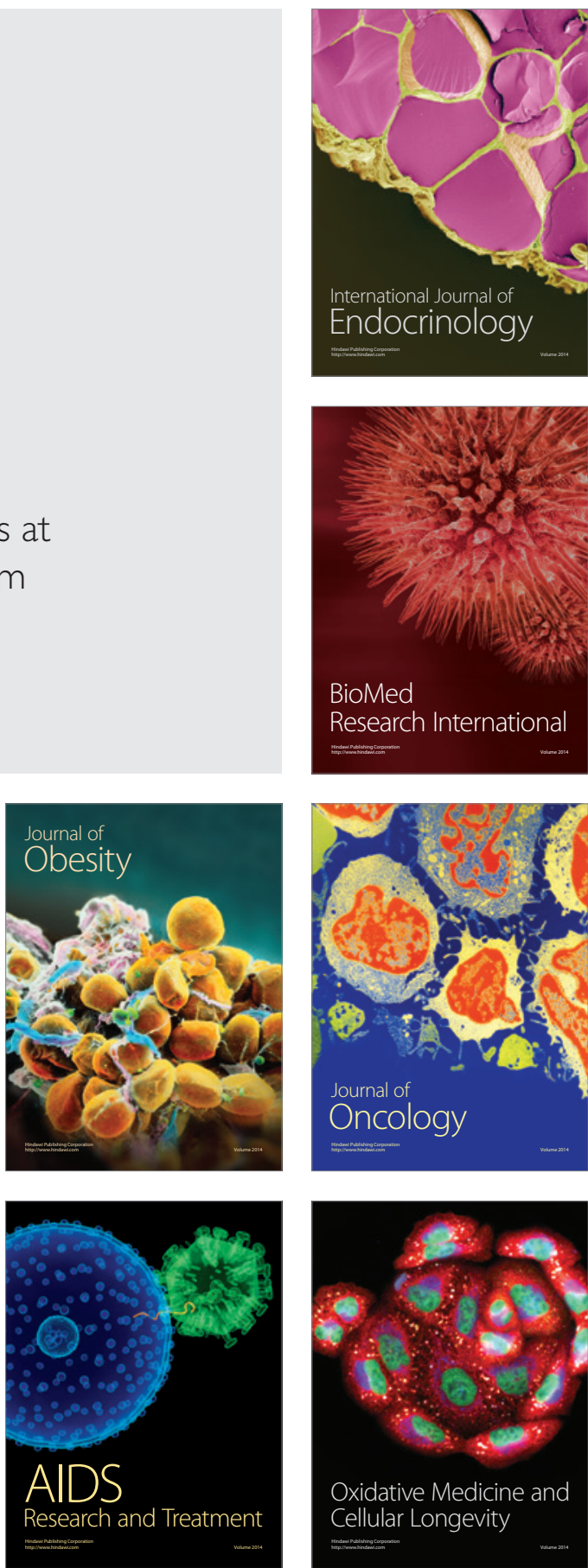